\title{
Postoperative diastolic perfusion pressure is associated with the development of acute kidney injury in patients after cardiac surgery: a retrospective analysis
}

Jifu Jin ${ }^{1,2,3,4+}$, Jiawei $Y u^{1,2,3,4 \dagger}$, Su Chi Chang ${ }^{5 \dagger}$, Jiarui $X u^{1,2,3,4}$, Sujuan $X u^{1,2,3,4}$, Wuhua Jiang ${ }^{1,2,3,4}$, Bo Shen ${ }^{1,2,3,4}$, Yamin Zhuang ${ }^{6}$, Chunsheng Wang ${ }^{7}$, Xiaogiang Ding ${ }^{1,2,3,4,8}$ and Jie Teng ${ }^{1,2,3,4,8^{*}}$

\begin{abstract}
Background: We aimed to investigate the relationship between the perioperative hemodynamic parameters and the occurrence of cardiac surgery-associated acute kidney injury.

Methods: A retrospective study was performed in patients who underwent cardiac surgery at a tertiary referral teaching hospital. Acute kidney injury was determined according to the KDIGO criteria. We investigated the association between the perioperative hemodynamic parameters and cardiac surgery-associated acute kidney injury to identify the independent hemodynamic predictors for acute kidney injury. Subgroup analysis was further performed in patients with chronic hypertension.

Results: Among 300 patients, 29.3\% developed acute kidney injury during postoperative intensive care unit period. Multivariate logistic analysis showed the postoperative nadir diastolic perfusion pressure, but not mean arterial pressure, central venous pressure and mean perfusion pressure, was independently linked to the development of acute kidney injury after cardiac surgery (odds ratio $0.945, P=0.045)$. Subgroup analyses in hypertensive subjects $(n=91)$ showed the postoperative nadir diastolic perfusion pressure and peak central venous pressure were both independently related to the development of acute kidney injury (nadir diastolic perfusion pressure, odds ratio 0.886, $P=0.033$; peak central venous pressure, odds ratio $1.328, P=0.010$, respectively).

Conclusions: Postoperative nadir diastolic perfusion pressure was independently associated with the development of cardiac surgery-associated acute kidney injury. Furthermore, central venous pressure should be considered as a potential hemodynamic target for hypertensive patients undergoing cardiac surgery.
\end{abstract}

Keywords: Diastolic perfusion pressure, Central venous pressure, Cardiac surgery, Acute kidney injury

\section{Background}

Acute kidney injury (AKI) is a frequent and severe complication after cardiac surgery, which has shown to be related to the increased morbidity, mortality and resource utilization [1-4]. The Acute Kidney Injury-Epidemiologic Prospective Investigation (AKI-EPI) study revealed the incidence of AKI using the complete Kidney Disease:

\footnotetext{
* Correspondence: teng.jie@zs-hospital.sh.cn

†jifu Jin, Jiawei Yu and Su Chi Chang contributed equally to this work.

'Department of Nephrology, Zhongshan Hospital, Shanghai Medical College,

Fudan University, 180 Fenglin Road, Shanghai 200032, China

${ }^{2}$ Shanghai Medical Center of Kidney Disease, Shanghai, China

Full list of author information is available at the end of the article
}

Improving Global Outcomes (KDIGO) criteria in critically ill patients was $57.3 \%[5,6]$. The cause of AKI is multifactorial and not completely understood yet. Multiple studies have identified risk factors for CSA-AKI that cannot be modified, including age, gender, baseline kidney function, diabetes mellitus and hypertension [6].

However, hemodynamic parameters, as modifiable risk factors, may be related to the progression of AKI in high-risk patients [7-11]. A number of studies have reported that an increased central venous pressure (CVP) is linked to the venous congestion and deterioration of kidney function in patients with cardiovascular disease

(c) The Author(s). 2019 Open Access This article is distributed under the terms of the Creative Commons Attribution 4.0 International License (http://creativecommons.org/licenses/by/4.0/), which permits unrestricted use, distribution, and 
and sepsis [12-14]. In addition, perfusion pressure - defined as the difference between arterial pressure and CVP - has been shown to increase the risk of developing AKI [15-17].

To date, a paucity of research has extensively studied the potential links between the systemic hemodynamic parameters, including postoperative extreme values (peak and nadir) of CVP, mean perfusion pressure (MPP), systolic perfusion pressure (SPP), diastolic perfusion pressure (DPP), and the development of AKI in cardiac surgery patients.

Accordingly, we investigated potential relationships between profound hemodynamic parameters (including MAP, CVP, DPP, MPP, and SPP) and CSA-AKI to establish if patients with AKI exhibited a lower perfusion pressure and higher CVP compared to patients not suffering from AKI.

\section{Methods}

\section{Patients}

We included all patients admitted in our ICU after cardiac surgery for a period of 3-months (June 1stSeptemper 1st 2015). The Institutional Ethics Committee of Zhongshan Hospital granted permission to study design and data collection, and the need for informed consents were waived because of the noninterventional design of the study.

\section{Inclusion and exclusion criteria}

The inclusion criteria were: adult patients (age $\geq 18$ years) who underwent elective cardiac surgery with or without $\mathrm{CPB}$ and stayed in the intensive care unit (ICU) for $\geq 24 \mathrm{~h}$ following surgery. Exclusion criteria were: patients who underwent cardiac transplantation or emergency operations; patients who had a history of previous renal replacement therapy or kidney transplantation; patients who were deceased $\leq 24 \mathrm{~h}$ after they were admitted to the ICU.

\section{Hemodynamic parameters}

Baseline blood pressure (BP) were measured at least three times prior to surgery and averaged for estimation of baseline hemodynamic values. Baseline MAP was estimated from diastolic arterial pressure (DAP) and systolic arterial pressure (SAP) using the standard formula: $\mathrm{MAP}=\mathrm{DAP}+$ (SAP - DAP) / 3.

Intraoperative $\mathrm{BP}$ values were measured every $10 \mathrm{~min}$ during the surgical period using a radial artery line, which was routinely positioned for hemodynamic monitoring. Time-weighted average (TWA) of intraoperative MAP values during CPB period were estimated as well. Systolic blood pressure values $>299 \mathrm{mmHg}$ or $<25$ $\mathrm{mmHg}$ were regarded as errors and excluded from the analysis as in the previous study [18].

Differences between the baseline MAP and intraoperative TWA-MAP were presented as absolute delta MAP (baseline MAP - intraoperative TWA-MAP) as well as relative delta MAP (absolute delta MAP/baseline MAP $\times 100 \%$ ), respectively.

Postoperative BP values were measured every 15 min for $24 \mathrm{~h}$ during the ICU period, and postoperative TWA-MAP was estimated over $24 \mathrm{~h}$ during such period. Given that hemodynamic parameters variates during the initial phase of ICU admission, the upper limits of the range (ULR) and lower limits of the range (LLR) over the first $24 \mathrm{~h}$ were recorded as well. The ULR of each hemodynamic parameter was defined as the highest value achieved within $24 \mathrm{~h}$ whereas the LLR was defined as the lowest value achieved within $24 \mathrm{~h}$ after ICU admission. The ULR and LLR of CVP within $24 \mathrm{~h}$ after surgery were also measured. Postoperative MPP was then calculated from postoperative TWAMAP within $24 \mathrm{~h}$ and extrema (ULR or LLR) values of CVP $(\mathrm{MPP}=$ MAP $-\mathrm{CVP})$. Similarly, postoperative DPP and SPP were calculated from postoperative TWA-DAP or TWA-SAP and extrema (ULR or LLR) values of CVP, respectively $(\mathrm{DPP}=\mathrm{DAP}-\mathrm{CVP}$; SPP $=\mathrm{SAP}-\mathrm{CVP}$; respectively) [17].

\section{Other variables}

An estimate of the glomerular filtration rate (eGFR) was calculated by the Modification of Diet in Renal Disease (MDRD) formula based on the patient characteristics and baseline serum creatinine, which was defined as the latest measurement prior to surgery. Serum creatinine was measured at least once a day during the postoperative ICU period as a standard of care. All of the subjects were separated into two groups depending on whether they suffered from AKI, defined according to the criteria published in the KDIGO guidelines [19].

Demographic data including gender, age, body weight, height, comorbidities and surgery type, surgical variables, vasopressin administration, fluid administration, erythrocytes transfusion, plasma transfusion, estimated intraoperative blood loss, intraoperative and postoperative urine output, mechanical ventilation time, length of ICU stay, and hospitalization time were obtained from the anesthetic database or electronic medical records.

\section{Statistical analysis}

Continuous data were shown as means \pm standard deviation or as a median (interquartile range) whereas categorical data were presented as numbers (\%). Potential differences between continuous variables were assessed using a $t$-test or Mann-Whitney U-test whereas changes in categorical variables were compared using Fisher's exact test or chisquared tests in patients with or without CSA-AKI.

Patient characteristics, intraoperative variables and postoperative variables were compared using the univariable logistic regression analysis to determine the risk factors for CSA-AKI. We calculated the odds ratio (OR) with 95\% 
confidence interval $(\mathrm{CI})$ for the predictors for AKI. The multivariable logistic regression analysis was further performed using the forward stepwise selection for the hemodynamic variables with a $P$ value of $<0.05$ considered to be predictive for CSA-AKI. Given that CPB duration and cross-clamp time were colinear factors, we enrolled only $\mathrm{CPB}$ duration in the multivariable analysis. After adjusting for the confounding factors (age, gender, baseline serum creatinine, baseline left ventricular ejection fraction [LVEF], CPB duration, fluid balance, and positive end-expiratory pressure $[\mathrm{PEEP}]$ ) for the occurrence of AKI, we established independent hemodynamic predictors for AKI development. Subgroup analyses were further performed in hypertensive subjects to establish the independent hemodynamic risk factors for CSA-AKI. Statistical significance was considered to be a two-tailed $P$ value $\leq 0.05$. All of the analyses were conducted using SPSS Statistics for Windows (ver. 18.0, SPSS Inc., US).

\section{Results}

\section{Basic characteristics}

In total, 300 cardiac surgery patients (173 men, mean age 54.5 years) were enrolled in our study. Eighty-eight (29.3\%) patients developed CSA-AKI based on the KDIGO criteria (25.0, 2.7 and 1.6\% in stages 1 to 3, respectively). Baseline serum creatinine levels were significantly higher in AKI patients when compared to non-AKI patients $(85.2 \pm$ $27.0 \mu \mathrm{mol} / \mathrm{L}$ vs. $76.7 \pm 19.6 \mu \mathrm{mol} / \mathrm{L} ; P=0.003)$. Acute Physiology and Chronic Health Evaluation III (APACHE III) score, EuroSCORE II at ICU admission, and surgery types were comparable in different patient groups. CPB duration as well as cross-clamp time in AKI group was remarkably longer than non-AKI group $(108.4 \pm 37.1 \mathrm{~min}$ vs. $90.1 \pm$ $31.8 \mathrm{~min}, P<0.05 ; 66.7 \pm 25.6 \mathrm{~min}$ vs. $55.8 \pm 24.9 \mathrm{~min}, P<$ $0.05)$. The length of ICU stay and total hospitalization time were statistically longer in AKI patients (both $P<0.05$ ). Inhospital mortality rate was significantly higher in AKI patients $(5.7 \%$ vs. $1.4 \%, P=0.037$ ) (Table 1$)$.

\section{Hemodynamic indices and CSA-AKI}

Table 2 shows a comparison of the perioperative hemodynamic parameters in AKI and non-AKI groups. Premorbid baseline BP (including SAP, DAP and MAP) levels were virtually identical in both groups. However, the patients with AKI had greater values of relative $\triangle \mathrm{MAP}$ $(25.3 \pm 9.4 \%$ vs. $22.5 \pm 11.0 \% ; P=0.039)$, and higher ULR and LLR values of postoperative CVP $(10.7 \pm 3.5 \mathrm{mmHg}$ vs. $9.8 \pm 2.7 \mathrm{mmHg} ; P=0.013 ; 5.0 \pm 2.2 \mathrm{mmHg}$ vs. $4.5 \pm 1.8$ mmHg; $P=0.048$, respectively) in comparison to the nonAKI patients. During the postoperative period, TWA-MAP at $6 \mathrm{~h}$ and $12 \mathrm{~h}$ was significantly reduced in the AKI groups (all $P<0.05$ ). In addition, ULR and LLR values of DPP as well as LLR value of MPP in AKI patients were statistically less than non-AKI patients $(51.9 \pm 7.1 \mathrm{mmHg}$ vs. $53.7 \pm 6.0$
mmHg; $P=0.027 ; 46.1 \pm 7.1 \mathrm{mmHg}$ vs. $48.4 \pm 6.4 \mathrm{mmHg}$; $P=0.008 ; 65.1 \pm 6.8 \mathrm{mmHg}$ vs. $66.7 \pm 6.1 \mathrm{mmHg}, P=0.037$, respectively) (Table 2).

\section{Risk factors for AKI development}

Univariate analyses showed that of the perioperative hemodynamic parameters measured, the relative change of $\triangle \mathrm{MAP}$, the ULR and LLR values of CVP and DPP, and the LLR value of MPP were implicated in AKI development (all $P<0.05$ ) (Table 3). Nevertheless, after adjusting confounding risk factors for the development of CSA-AKI (including age, gender, baseline serum creatinine, baseline LVEF, and CPB duration, fluid balance, PEEP), only the LLR value of DPP was significantly related to the AKI occurrence (odds ratio [OR] for $1 \mathrm{mmHg}$ increase $=0.945$; 95\% CI 0.894-0.999; $P=0.045$ ) (Table 4).

\section{Subgroup analyses in patients with chronic hypertension} Subgroup analysis was further carried out in cardiac surgery patients with chronic hypertension $(n=91)$. After adjusting for risk factors for AKI (age, gender, baseline serum creatinine, baseline LVEF, CPB duration, fluid balance, average PEEP), the ULR value of CVP and the LLR value of DPP were both significantly and independently associated with AKI occurrence (OR for $1 \mathrm{mmHg}$ increase of CVP $[\mathrm{ULR}]=1.328,95 \% \mathrm{CI}: 1.070-1.648$, $P=0.010$; OR for $1 \mathrm{mmHg}$ increase of DPP [LLR] $=$ 0.886, 95\% CI: 0.793-0.990, $P=0.010$ ) (Table 5).

\section{Discussion}

Our study revealed that in patients who underwent cardiac surgery, the nadir value of postoperative DPP within $24 \mathrm{~h}$ was independently related to the development of AKI. Subgroup analyses further implied that postoperative nadir DPP and peak CVP were both closely linked to AKI development in patients with chronic hypertension.

Several studies have reported that there was no significant association between intraoperative MAP and postoperative AKI $[20,21]$. However, the MAP deficit, defined as the difference between the premorbid and intraoperative MAP, was found to be related to the occurrence of AKI [22]. Univariate analysis of our data revealed the relative change of MAP deficit, but not the absolute MAP deficit, was closely linked to the development of CSA-AKI. However, when after adjustment for the risk factors for AKI, there was no relationship between MAP deficit (both relative and absolute) and the occurrence of AKI.

Recently, several studies have demonstrated that MPP or DPP was related to the development or progression of AKI. In a retrospective study of critically ill patients, it was reported that patients with an MPP $\leq 59 \mathrm{mmHg}$ had a higher risk of AKI progression [23]. Moreover, the median value of MPP deficit [=\%(baseline MPP-achieved $\mathrm{MPP}$ /baseline MPP] was higher in patients with severe 
Table 1 Patient characteristics according to the presence or absence of $A K$

\begin{tabular}{|c|c|c|c|}
\hline & Non-AKI & AKI & $P$-value \\
\hline Total & $212(70.7)$ & $88(29.3)$ & - \\
\hline Male (\%) & $102(48.1)$ & $71(80.7)$ & $<0.001$ \\
\hline Age (years) & $52.9 \pm 14.3$ & $58.1 \pm 12.1$ & 0.002 \\
\hline BMI $\left(\mathrm{kg} / \mathrm{m}^{2}\right)$ & $22.9 \pm 3.1$ & $24.1 \pm 3.7$ & 0.090 \\
\hline \multicolumn{4}{|l|}{ Comorbidities } \\
\hline CKD (\%) & $10(4.7)$ & $10(11.4)$ & 0.036 \\
\hline Hypertension (\%) & $57(26.9)$ & 34 (38.6) & 0.044 \\
\hline Diabetes (\%) & $17(8.0)$ & $11(12.5)$ & 0.224 \\
\hline Cerebrovascular disease (\%) & $11(5.2)$ & $3(3.4)$ & 0.506 \\
\hline Atrial fibrillation (\%) & $40(18.9)$ & $17(19.3)$ & 0.928 \\
\hline Hyperlipidemia (\%) & $6(2.8)$ & $2(2.3)$ & 0.785 \\
\hline \multicolumn{4}{|l|}{ Medication before admission } \\
\hline Aspirin (\%) & $31(14.6)$ & $8(9.1)$ & 0.195 \\
\hline CCB (\%) & $14(6.6)$ & $10(11.4)$ & 0.166 \\
\hline ACEI (\%) & $9(4.2)$ & $1(1.1)$ & 0.172 \\
\hline ARB (\%) & $11(3.7)$ & $4(1.3)$ & 0.816 \\
\hline Diuretics (\%) & $6(2.0)$ & $5(1.7)$ & 0.231 \\
\hline$\beta$-blocker (\%) & $15(7.1)$ & $6(6.8)$ & 0.937 \\
\hline Statins (\%) & $13(6.1)$ & $1(1.1)$ & 0.062 \\
\hline Preoperative contrast exposure(\%) & $118(55.7)$ & $51(58.0)$ & 0.715 \\
\hline $\begin{array}{l}\text { Intervals between contrast exposure } \\
\text { and surgery (days) }\end{array}$ & $3.52 \pm 2.73$ & $3.34 \pm 2.58$ & 0.683 \\
\hline \multicolumn{4}{|l|}{ Baseline kidney function } \\
\hline eGFR (MDRD) & $89.1 \pm 17.6$ & $85.7 \pm 19.1$ & 0.146 \\
\hline Serum creatinine $(\mu \mathrm{mol} / \mathrm{L})$ & $76.7 \pm 19.6$ & $85.2 \pm 27.0$ & 0.003 \\
\hline LVEF & $62.7 \pm 6.9$ & $60.0 \pm 8.8$ & 0.006 \\
\hline NYHA III-IV (\%) & $135(63.7)$ & $57(64.8)$ & 0.857 \\
\hline Baseline hemoglobin ( $g / L)$ & $130.2 \pm 17.2$ & $133.2 \pm 15.7$ & 0.166 \\
\hline APACHE III & $12.6 \pm 3.4$ & $13.0 \pm 4.0$ & 0.464 \\
\hline EuroSCORE II & $1.20 \pm 0.71$ & $1.35 \pm 0.89$ & 0.161 \\
\hline \multicolumn{4}{|l|}{ Surgery type } \\
\hline CABG (\%) & $36(17.1)$ & $10(11.4)$ & 0.142 \\
\hline Single valve surgery (\%) & $75(35.5)$ & $34(38.6)$ & 0.455 \\
\hline Combined valve surgery (\%) & $49(23.3)$ & $22(25.0)$ & 0.858 \\
\hline CABG + valve surgery (\%) & $4(1.9)$ & $3(3.4)$ & 0.419 \\
\hline Aortic root surgery (\%) & $47(22.3)$ & $20(22.7)$ & 0.941 \\
\hline \multicolumn{4}{|l|}{ Intraoperative fluid balance } \\
\hline Estimated blood loss (ml) & $414 \pm 313$ & $462 \pm 393$ & 0.358 \\
\hline Ultrafiltration (ml) & $2295 \pm 794$ & $2525 \pm 800$ & 0.092 \\
\hline Erythrocytes transfusion (ml) & $420 \pm 215$ & $470 \pm 289$ & 0.144 \\
\hline FFP transfusion (ml) & $386 \pm 133$ & $531 \pm 222$ & 0.005 \\
\hline \multicolumn{4}{|l|}{ Surgery variables } \\
\hline CPB duration (min) & $90.1 \pm 31.8$ & $108.4 \pm 37.1$ & $<0.001$ \\
\hline Cross-clamp duration (min) & $55.8 \pm 24.9$ & $66.7 \pm 25.6$ & 0.004 \\
\hline
\end{tabular}

Table 1 Patient characteristics according to the presence or absence of AKI (Continued)

\begin{tabular}{llll}
\hline & Non-AKl & AKI & P-value \\
\hline Dose of vasopressor or inotropes & & & \\
Norepinephrine (mg) & $6.2 \pm 5.7$ & $10.2 \pm 13.8$ & 0.003 \\
Epinephrine (mg) & $0.4 \pm 1.3$ & $1.2 \pm 2.6$ & 0.021 \\
Dopamine (mg) & $80.6 \pm 112.1$ & $86.6 \pm 97.2$ & 0.721 \\
Dobutamine (mg) & $54.7 \pm 72.0$ & $66.1 \pm 82.2$ & 0.322 \\
Fluid balance at POD ${ }_{24 h}(\mathrm{ml})$ & $-290 \pm$ & $-256 \pm$ & 0.876 \\
& 1475 & 1752 & \\
Fluid infusion (ml) & $3804 \pm 850$ & $3841 \pm 844$ & 0.754 \\
Fluid output (ml) & $4157 \pm$ & $4325 \pm$ & 0.474 \\
& 1520 & 1514 & \\
Urine output (ml) & $2099 \pm 721$ & $1789 \pm 734$ & 0.002 \\
Outcomes & & & \\
CRRT (\%) & $0(0.0)$ & $5(5.7)$ & 0.037 \\
LOS in ICU (hours) & $34.8 \pm 29.3$ & $47.7 \pm 30.8$ & 0.001 \\
LOS in hospital (days) & $11.0 \pm 2.8$ & $12.1 \pm 4.1$ & 0.027 \\
In-hospital mortality (\%) & $3(1.4)$ & $5(5.7)$ & 0.037
\end{tabular}

The data in the table are expressed as mean \pm standard deviation or number (\%). Continuous variables were compared using the $\mathrm{T}$ test, whereas categorical variables were compared using chi-squared test

septic AKI when compared to those without this condition $[16,17]$. In cardiovascular surgery patients who were vasopressor-dependent, it was demonstrated that deficits in DPP and MPP were significantly related to the progression of AKI [15]. Similarly, we found that postoperative peak and nadir values of DPP and the nadir value of MPP were significantly reduced in patients with AKI. However, after adjustment for confounding factors for AKI, only the nadir value of DPP was independently related to the development of AKI. The difference of DPP between AKI and non-AKI patients in our study was greater than the results from previous studies [15]. Furthermore, given that postoperative MPP levels in our cohort were relatively higher than that in previous studies, no independent associations were detected between postoperative MPP and the development of AKI from our analyses.

Subgroups analyses indicated that the peak value of postoperative CVP also independently contributed to the development of AKI in patients with chronic hypertension. The link between AKI and CVP was most notable in patients with cardiovascular disease [12, 13]. More recently, Williams et al. reported that in cardiovascular surgery patients for CVP increments $>5 \mathrm{mmHg}$ above the $9 \mathrm{mmHg}$ threshold, the risk of developing AKI had an odds ratio of 1.3 [11]. Yang et al. proposed that high CVP value (especially higher than $10 \mathrm{cmH}_{2} \mathrm{O}$ ) at the end of cardiac surgery was independently associated with AKI [24]. Increments of CVP, especially in hypervolemic status, impedes venous return, which results in elevated venous pressure and further decreased cardiac output. 
Table 2 Perioperative hemodynamic parameters and AKI

\begin{tabular}{|c|c|c|c|}
\hline & Non-AKI & AKI & $\begin{array}{l}P \text { - } \\
\text { value }\end{array}$ \\
\hline \multicolumn{4}{|l|}{ Presurgical period } \\
\hline Baseline SAP (mmHg) & $124.6 \pm 15.8$ & $127.1 \pm 14.1$ & 0.202 \\
\hline Baseline DAP (mmHg) & $73.2 \pm 9.5$ & $72.1 \pm 10.4$ & 0.398 \\
\hline Baseline MAP (mmHg) & $90.3 \pm 9.6$ & $90.5 \pm 9.1$ & 0.894 \\
\hline \multicolumn{4}{|l|}{ Surgical period } \\
\hline TWA-MAP (mmHg) & $69.2 \pm 7.8$ & $67.7 \pm 8.3$ & 0.114 \\
\hline $\begin{array}{l}\triangle \mathrm{MAP} \text { relative to baseline } \\
(\mathrm{mmHg})\end{array}$ & $21.0 \pm 11.4$ & $23.4 \pm 10.0$ & 0.091 \\
\hline$\% \triangle$ MAP relative to baseline (\%) & $22.5 \pm 11.0$ & $25.3 \pm 9.4$ & 0.039 \\
\hline \multicolumn{4}{|l|}{ Postsurgical period } \\
\hline CVP (ULR) (mmHg) & $9.8 \pm 2.7$ & $10.7 \pm 3.5$ & 0.013 \\
\hline CVP (LLR) (mmHg) & $4.5 \pm 1.8$ & $5.0 \pm 2.2$ & 0.048 \\
\hline TWA-SAP (mmHg) & $113.3 \pm 8.6$ & $113.6 \pm 10.5$ & 0.835 \\
\hline TWA-DAP (mmHg) & $58.2 \pm 6.2$ & $56.8 \pm 6.9$ & 0.099 \\
\hline TWA-MAP (mmHg) & $76.5 \pm 5.8$ & $75.8 \pm 6.8$ & 0.313 \\
\hline $\mathrm{SPP}(\mathrm{ULR})(\mathrm{mmHg})$ & $108.8 \pm 8.7$ & $108.6 \pm 10.6$ & 0.859 \\
\hline SPP (LLR) (mmHg) & $103.5 \pm 9.0$ & $102.9 \pm 10.4$ & 0.581 \\
\hline DPP (ULR) (mmHg) & $53.7 \pm 6.0$ & $51.9 \pm 7.1$ & 0.027 \\
\hline DPP (LLR) (mmHg) & $48.4 \pm 6.4$ & $46.1 \pm 7.1$ & 0.008 \\
\hline MPP (ULR) (mmHg) & $72.1 \pm 5.7$ & $70.8 \pm 7.0$ & 0.112 \\
\hline MPP (LLR) (mmHg) & $66.7 \pm 6.1$ & $65.1 \pm 6.8$ & 0.037 \\
\hline
\end{tabular}

Continuous variables are expressed as mean \pm standard deviation and compared using the $T$ test
Table 3 Univariate analysis with AKI as the outcome variable

\begin{tabular}{llll}
\hline Predictor & Odds Ratio & $95 \% \mathrm{Cl}$ & -value \\
\hline Male/Sex (present) & 4.504 & $2.487-8.157$ & $<0.001$ \\
Age (per 1 year increase) & 1.030 & $1.009-1.050$ & 0.004 \\
BMI (per 1 unit increase) & 1.109 & $0.983-1.252$ & 0.092 \\
Hypertension (present) & 1.712 & $1.012-2.896$ & 0.045 \\
LVEF (per 1 unit increase) & 0.956 & $0.926-0.988$ & 0.008 \\
Baseline SCr (per 1 $\mu$ mol/L & 1.017 & $1.005-1.030$ & 0.005 \\
increase) & & & \\
CPB (per 1 min increase) & 1.015 & $1.007-1.024$ & 0.001 \\
Cross-clamp (per 1 min increase) & 1.017 & $1.005-1.029$ & 0.005 \\
\% $\triangle M A P$ relative to baseline & 1.026 & $1.001-1.051$ & 0.040 \\
(per 1 unit increase) & & & \\
CVP (ULR) (per 1 mmHg increase) & 1.113 & $1.022-1.211$ & 0.014 \\
CVP (LLR) (per 1 mmHg increase) & 1.132 & $1.000-1.281$ & 0.050 \\
DPP (ULR) (per 1 mmHg increase) & 0.956 & $0.918-0.995$ & 0.028 \\
DPP (LLR) (per 1 mmHg increase) & 0.949 & $0.913-0.987$ & 0.009 \\
MPP (LLR) (per 1 mmHg increase) & 0.959 & $0.921-0.998$ & 0.039 \\
\hline
\end{tabular}

Table 4 Multivariable logistic analysis with AKI as the outcome variable

\begin{tabular}{llll}
\hline Predictor & Odds Ratio $^{a}$ & $95 \% \mathrm{Cl}$ & P-value \\
\hline $\begin{array}{l}\text { \% } \triangle \text { MAP relative to baseline } \\
\text { (per 1 unit increase) }\end{array}$ & 1.002 & $0.969-1.037$ & 0.907 \\
$\begin{array}{l}\text { CVP (ULR) (per 1 mmHg } \\
\text { increase) }\end{array}$ & 1.113 & $0.997-1.242$ & 0.057 \\
CVP (LLR) (per $1 \mathrm{mmHg}$ increase) & 1.160 & $0.988-1.362$ & 0.071 \\
DPP (ULR) (per $1 \mathrm{mmHg}$ increase) & 0.949 & $0.895-1.007$ & 0.082 \\
DPP (LLR) (per 1 mmHg increase) & 0.945 & $0.894-0.999$ & 0.045 \\
MPP (LLR) (per 1 mmHg increase) & 0.951 & $0.899-1.005$ & 0.076 \\
\hline
\end{tabular}

Furthermore, an increased venous pressure may trigger an increased renal interstitial pressure, which commonly leads to impaired microcirculatory flow and reduced glomerular filtration rate $[25,26]$. Hence, postoperative CVP levels should be closely monitored in patients undergoing cardiac surgery, especially in those who had a history of chronic hypertension.

As far as we know, the present research is the first to have investigated the relationship between perioperative extensive hemodynamic parameters and CSA-AKI. Based on our results, we suggest the nadir level of DPP may serve as a surrogate for indicating the risk of AKI development. In cardiac surgery patients with decreased DPP, excessive fluid resuscitation should be under deliberation in patients with chronic hypertension. Further interventional studies should be carried out to clarify whether potential therapeutic manipulations such as increasing DPP or limiting CVP may prevent AKI development in cardiac surgery patients.

However, our investigation still confers some limitations. First, it was a single-center retrospective study with a relatively small cohort of patients. Our study only enrolled patients who underwent cardiac surgery, limiting the applicability of our findings to general settings. Second, since preoperative CVP values were not available in our database, premorbid perfusion pressure was not taken into account in the analysis. Finally, a causal relationship between relevant

Table 5 Subgroup analysis with hypertension: adjusted association with AKI

\begin{tabular}{llll}
\hline Predictor & Odds Ratio $^{a}$ & $95 \% \mathrm{Cl}$ & P-value \\
\hline $\begin{array}{l}\text { \% } \triangle \text { MAP relative to baseline } \\
\text { (per 1 unit increase) }\end{array}$ & 0.991 & $0.924-1.062$ & 0.788 \\
CVP (ULR) (per 1 mmHg increase) & 1.328 & $1.070-1.648$ & 0.010 \\
CVP (LLR) (per $1 \mathrm{mmHg}$ increase) & 1.186 & $0.889-1.583$ & 0.245 \\
DPP (ULR) (per 1 mmHg increase) & 0.945 & $0.856-1.045$ & 0.272 \\
DPP (LLR) (per $1 \mathrm{mmHg}$ increase) & 0.886 & $0.793-0.990$ & 0.033 \\
MPP (LLR) (per 1 mmHg increase) & 0.908 & $0.813-1.014$ & 0.087 \\
\hline
\end{tabular}


hemodynamic variables and the development of AKI may not be established due to the inherent bias of the study design. Therefore, further interventional studies should be carried out to establish possible causal connections between hemodynamic parameters and AKI, which will be beneficial for the hemodynamic management in patients undergoing cardiac surgery.

\section{Conclusions}

Postoperative nadir DPP was independently associated with the development of AKI. Postoperative peak CVP was significant for AKI in patients with chronic hypertension. Further investigations are needed in the future to verify whether advanced hemodynamic management (specifically targeting DPP and CVP) may reduce the incidence of CSA-AKI.

\section{Abbreviations \\ AKI: Acute kidney injury; APACHE: Acute Physiology and Chronic Health Evaluation; BP: Blood pressure; Cl: Confidence interval; CPB: Cardiopulmonary bypass; CSA-AKI: Cardiac surgery-associated acute kidney injury; CVP: Central venous pressure; DAP: Diastolic arterial pressure; DPP: Diastolic perfusion pressure; eGFR: Estimated glomerular filtration rate; ICU: Intensive care unit; LLR: Lower limit of the range; LVEF: Left ventricular ejection fraction; MAP: Mean arterial pressure; MDRD: Modification of Diet in Renal Disease; MPP: Mean perfusion pressure; OR: Odds ratio; PEEP: Positive end-expiratory pressure; SAP: Systolic arterial pressure; SPP: Systolic perfusion pressure; TWA: Time-weighted average; ULR: Upper limit of the range}

\section{Acknowledgements}

Not applicable.

\section{Authors' contributions}

$J J, J Y, S C C$, and JX were responsible for the study design, data acquisition and analysis. JJ, SX, WJ, and BS drafted the first manuscript. YW, YZ, CW, XD, and JT conducted a critical revision of manuscript. All authors read and approved the final manuscript.

\section{Funding}

This study was supported by the research grants from the Science and Technology Commission of Shanghai Municipality (14DZ2260200), Shanghai Clinical Medical Center for Kidney Disease Project support by Shanghai Municipal Health Commission (2017ZZ01015), Shanghai Municipal Hospital Frontier Technology Project supported by Shanghai ShenKang Hospital Development Center (SHDC12018127), and the Xiamen Science and Technology Plan in 2018 (3502Z20184009). These funding have no role in the design of study, data collection, analysis and neither interpretation of data nor writing of the manuscript.

\section{Availability of data and materials}

The datasets used and/or analyzed during the current study are available from the corresponding author on reasonable request.

\section{Ethics approval and consent to participate}

The study was approved by the Institutional Ethics Committee of Zhongshan Hospital, Fudan University, Shanghai, China (B2018-175). The need for informed consents were waived by the Institutional Ethics Committee because this was a retrospective analysis of data collected prospectively with no breach of privacy or anonymity.

\section{Consent for publication}

Not applicable.

\section{Competing interests}

The authors declare that they have no competing interests.

\section{Author details}

${ }^{1}$ Department of Nephrology, Zhongshan Hospital, Shanghai Medical College, Fudan University, 180 Fenglin Road, Shanghai 200032, China. ${ }^{2}$ Shanghai Medical Center of Kidney Disease, Shanghai, China. ${ }^{3}$ Shanghai Institute of Kidney and Dialysis, Shanghai, China. ${ }^{4}$ Shanghai Key Laboratory of Kidney and Blood Purification, Shanghai, China. ${ }^{5}$ Department of Cardiology, Zhongshan Hospital, Shanghai Medical College, Fudan University, Shanghai, China. ${ }^{6}$ Department of Intensive Care Medicine, Zhongshan Hospital, Shanghai Medical College, Fudan University, Shanghai, China. ${ }^{7}$ Department of Cardiovascular Surgery, Zhongshan Hospital, Shanghai Medical College, Fudan University, Shanghai, China. ${ }^{8}$ Department of Nephrology, Xiamen Branch, Zhongshan Hospital, Fudan University, Xiamen, Fujian, China.

Received: 19 August 2019 Accepted: 20 November 2019

Published online: 10 December 2019

\section{References}

1. Chertow GM, Burdick E, Honour M, Bonventre JV, Bates DW. Acute kidney injury, mortality, length of stay, and costs in hospitalized patients. J Am Soc Nephrol. 2005;16:3365-70.

2. Elmistekawy E, McDonald B, Hudson C, Ruel M, Mesana T, Chan V, et al. Clinical impact of mild acute kidney injury after cardiac surgery. Ann Thorac Surg. 2014;98:815-22.

3. Hobson CE, Yavas S, Segal MS, Schold JD, Tribble CG, Layon AJ, et al. Acute kidney injury is associated with increased long-term mortality after cardiothoracic surgery. Circulation. 2009;119:2444-53.

4. Liangos O, Wald R, O'bell JW, Price L, Pereira BJ, Jaber BL. Epidemiology and outcomes of acute renal failure in hospitalized patients: a national survey. Clin J Am Soc Nephrol. 2006;1:43-51.

5. Hoste EA, Bagshaw SM, Bellomo R, Cely CM, Colman R, Cruz DN, et al. Epidemiology of acute kidney injury in critically ill patients: the multinational AKI-EPI study. Intensive Care Med. 2015;41(8):1411-23.

6. Brown JR, Cochran RP, Leavitt BJ, Dacey LJ, Ross CS, MacKenzie TA, et al. Multivariable prediction of renal insufficiency developing after cardiac surgery. Circulation. 2007;116:1139-43.

7. Cheung AT. Exploring an optimum intra/postoperative management strategy for acute hypertension in the cardiac surgery patient. J Card Surg. 2006;21:S8-S14.

8. Gambardella I, Gaudino M, Ronco C, Lau C, Ivascu N, Girardi LN. Congestive kidney failure in cardiac surgery: the relationship between central venous pressure and acute kidney injury. Interact Cardiovasc Thorac Surg. 2016;23:800-5.

9. Haase M, Bellomo R, Story D, Letis A, Klemz K, Matalanis G, et al. Effect of mean arterial pressure, haemoglobin and blood transfusion during cardiopulmonary bypass on post-operative acute kidney injury. Nephrol Dial Transplant. 2012;27:153-60.

10. Sickeler R, Phillips-Bute B, Kertai MD, Schroder J, Mathew JP, Swaminathan $M$, et al. The risk of acute kidney injury with co-occurrence of anemia and hypotension during cardiopulmonary bypass relative to anemia alone. Ann Thorac Surg. 2014:97:865-71.

11. Williams JB, Peterson ED, Wojdyla D, Harskamp R, Southerland KW, Ferguson $T B$, et al. Central venous pressure after coronary artery bypass surgery: does it predict postoperative mortality or renal failure? J Crit Care. 2014;29:1006-10.

12. Damman K, Navis G, Smilde TD, Voors AA, van der Bij W, van Veldhuisen DJ, et al. Decreased cardiac output, venous congestion and the association with renal impairment in patients with cardiac dysfunction. Eur J Heart Fail. 2007;9:872-8.

13. Mullens W, Abrahams Z, Francis GS, Sokos G, Taylor DO, Starling RC, et al. Importance of venous congestion for worsening of renal function in advanced decompensated heart failure. J Am Coll Cardiol. 2009;53:589-96.

14. Legrand M, Dupuis C, Simon C, Gayat E, Mateo J, Lukaszewicz AC, et al. Association between systemic hemodynamics and septic acute kidney injury in critically ill patients: a retrospective observational study. Crit Care. 2013;17:R278.

15. Saito S, Uchino S, Takinami M, Uezono S, Bellomo R. Postoperative blood pressure deficit and acute kidney injury progression in vasopressordependent cardiovascular surgery patients. Crit Care. 2016;20:74.

16. Panwar R, Lanyon N, Davies AR, Bailey M, Pilcher D, Bellomo R. Mean perfusion pressure deficit during the initial management of shock--an observational cohort study. J Crit Care. 2013;28:816-24.

17. Wong BT, Chan MJ, Glassford NJ, Martensson J, Bion V, Chai SY, et al. Mean arterial pressure and mean perfusion pressure deficit in septic acute kidney injury. J Crit Care. 2015;30:975-81. 
18. Aronson S, Phillips-Bute B, Stafford-Smith M, Fontes M, Gaca J, Mathew JP, et al. The association of postcardiac surgery acute kidney injury with intraoperative systolic blood pressure hypotension. Anesthesiol Res Pract. 2013:2013:174091.

19. Kellum JA, Lameire N, KDIGO AKI. Guideline work group. Diagnosis, evaluation, and management of acute kidney injury: a KDIGO summary (part 1). Crit Care. 2013;17:204.

20. Azau A, Markowicz P, Corbeau J,, Cottineau C, Moreau X, Baufreton C, et al. Increasing mean arterial pressure during cardiac surgery does not reduce the rate of postoperative acute kidney injury. Perfusion. 2014;29:496-504.

21. Kandler K, Jensen ME, Nilsson JC, Moller CH, Steinbruchel DA. Arterial pressure during cardiopulmonary bypass is not associated with acute kidney injury. Acta Anaesthesiol Scand. 2015;59:625-31.

22. Kanji HD, Schulze CJ, Hervas-Malo M, Wang P, Ross DB, Zibdawi M, et al. Difference between pre-operative and cardiopulmonary bypass mean arterial pressure is independently associated with early cardiac surgeryassociated acute kidney injury. J Cardiothorac Surg. 2010;5:71.

23. Ostermann M, Hall A, Crichton S. Low mean perfusion pressure is a risk factor for progression of acute kidney injury in critically ill patients - a retrospective analysis. BMC Nephrol. 2017;18:151.

24. Yang Y, Ma J, Zhao L. High central venous pressure is associated with acute kidney injury and mortality in patients underwent cardiopulmonary bypass surgery. J Crit Care. 2018;48:211-5.

25. Prowle JR, Kirwan CJ, Bellomo R. Fluid management for the prevention and attenuation of acute kidney injury. Nat Rev Nephrol. 2014;10:37-47.

26. Vellinga NA, Ince C, Boerma EC. Elevated central venous pressure is associated with impairment of microcirculatory blood flow in sepsis: a hypothesis generating post hoc analysis. BMC Anesthesiol. 2013;13:17.

\section{Publisher's Note}

Springer Nature remains neutral with regard to jurisdictional claims in published maps and institutional affiliations.

Ready to submit your research? Choose BMC and benefit from:

- fast, convenient online submission

- thorough peer review by experienced researchers in your field

- rapid publication on acceptance

- support for research data, including large and complex data types

- gold Open Access which fosters wider collaboration and increased citations

- maximum visibility for your research: over $100 \mathrm{M}$ website views per year

At BMC, research is always in progress.

Learn more biomedcentral.com/submissions 